\title{
Conjugated polymer nanocomposites: Synthesis, dielectric, and microwave absorption studies
}

\author{
Anil Ohlan, ${ }^{1}$ Kuldeep Singh, ${ }^{1}$ Amita Chandra, ${ }^{2}$ V. N. Singh, ${ }^{3}$ and S. K. Dhawan ${ }^{1, a)}$ \\ ${ }_{1}^{1}$ Polymeric and Soft Materials Section, National Physical Laboratory, New Delhi 110012, India \\ ${ }^{2}$ Department of Physics and Astrophysics, University of Delhi, Delhi 110007, India \\ ${ }^{3}$ Department of Physics, Thin Film Laboratory, Indian Institute of Technology Delhi, New Delhi 110016, \\ India
}

(Received 2 February 2009; accepted 11 July 2009; published online 21 August 2009)

\begin{abstract}
Nanocomposites of polyaniline with barium ferrite and titanium dioxide $\left(\mathrm{TiO}_{2}\right)$ are synthesized via in situ emulsion polymerization. The transmission electron microscopy (TEM) and high resolution TEM result shows the formation of array of nanoparticles encapsulated within the polymer chains during the synthesis process. The high value of microwave absorption, $58 \mathrm{~dB}$ (>99.999\% attenuation) results from the combined effect of the nanoparticles and the polymer matrix. The amount of barium ferrite has the profound effect on permittivity $(\varepsilon)$, permeability $(\mu)$, and microwave absorption of the nanocomposite. The contribution to the absorption value comes mainly due the magnetic losses $\left(\mu^{\prime \prime}\right)$ in barium ferrite and dielectric losses $\left(\varepsilon^{\prime \prime}\right)$ in $\mathrm{TiO}_{2}$ and polyaniline. (c) 2009 American Institute of Physics. [DOI: 10.1063/1.3200958]
\end{abstract}

\section{INTRODUCTION}

An unwanted disturbance called electromagnetic interference (EMI) is one that affects an electrical circuit due to electromagnetic (EM) radiation emitted from an external source carrying rapidly changing electrical currents. ${ }^{1,2}$ The disturbance may interrupt, or otherwise degrade or limit the effective performance of the circuit. Intrinsic conducting polymers (ICPs) having extended $\pi$-conjugated system with conductivity in semiconductor regime has emerged as a potential class of materials for EMI shielding and microwave absorbers. ${ }^{3-6}$ To protect the electronic equipment for the commercial applications, the material with shielding effectiveness (SE) measured in decibel $(\mathrm{dB})$ greater than $30 \mathrm{~dB}$ should be adequate, while for military application, the requirement are significantly higher, in the range between 80 and $100 \mathrm{~dB}$. Here we have shown that nanocomposites of polyaniline with barium ferrite and titanium dioxide $\left(\mathrm{TiO}_{2}\right)$ named PBT composite possesses the high value of microwave absorption, $58 \mathrm{~dB}(\sim 99.9999 \%$ attenuation). While recently, ICP composites with ferrite, singlewalled carbon nanotube (CNT), and multiwalled CNT have been reported as EMI shielding material with $\mathrm{SE}$ of $\sim 30 \mathrm{~dB}$ having high thickness. $^{7-9}$ The higher SE of PBT results from the combined effect of the nanoparticles and the polymer matrix. The contribution to the microwave absorption comes mainly from the magnetic losses in barium ferrite and dielectric losses in $\mathrm{TiO}_{2}$ and polyaniline. The achievement of higher SE can lead them to be used as an additive in paints which acts as coating material and may replace the ferrite/metal coatings. Many research groups are working on this aspect of conjugated polymers, as unlike metals, they not only reflect the EM radiation but also absorb them. ${ }^{10}$ The microwave absorbing properties are determined by the complex relative per-

\footnotetext{
a) Tel.: +91-11-45609401. FAX: +91-11-25726938. Electronic mail skdhawan@mail.nplindia.ernet.in.
}

mittivity $\left(\varepsilon_{r}=\varepsilon^{\prime}-j \varepsilon^{\prime \prime}\right)$, permeability $\left(\mu_{r}=\mu^{\prime}-j \mu^{\prime \prime}\right)$ and the microstructure of the absorber. Technical requirement for the absorber limits the number of ferromagnetic materials that can be used in the microwave range above few gigahertz. ${ }^{11}$ However, nanosized ferromagnetic particles have properties that can be varied with the size ${ }^{12}$ and are different from those of the bulk materials. At high frequency, the permeability of the magnetic material decreases due to the eddy current losses developed by the EM wave. Therefore, it is better to use conducting matrix to suppress the eddy current phenomenon to enhance the effective interaction with the absorber. ${ }^{13}$ Among the EM wave absorbers, the composites of magnetic particles in the insulating matrix have been extensively studied. Che et al. ${ }^{14}$ reported the synthesis of CNT encapsulated with Fe nanoparticles showing good absorption behavior but the complex synthesis of CNT filled with magnetic nanoparticles is not favorable for practical applications. Abbas et $a l .{ }^{15}$ reported that the microwave absorption properties of barium hexaferrite and its polymer composite show good attenuation value of $30 \mathrm{~dB}$ in the $X$-band but the synthesis process is mainly governed by mixing the ferrite with the polymer. Moreover, thick samples are required for higher attenuation.

\section{EXPERIMENTAL}

The synthesis of barium ferrite has been carried out via precursor route ${ }^{16}$ by dissolving 1:12:13 molar ratio of barium nitrate, ferric nitrate, and citric acid, respectively, in distilled water. The barium ferrite and $\mathrm{TiO}_{2}$ were further grinded for $6 \mathrm{~h}$ using Retsch "PM-400" planetary-ball mill in tungsten carbide jars. The formation of barium ferrite and $\mathrm{TiO}_{2}$ phase has been confirmed by x-ray diffractometer. The resulting nanosized barium ferrite along with $\mathrm{TiO}_{2}$ nanoparticles have been homogenized in $0.3 \mathrm{M}$ aqueous solution of dodecyl benzene sulfonic acid (DBSA) to form a whitish brown emulsion solution. Appropriate amount of aniline $(0.1 M)$ has been 
(a)
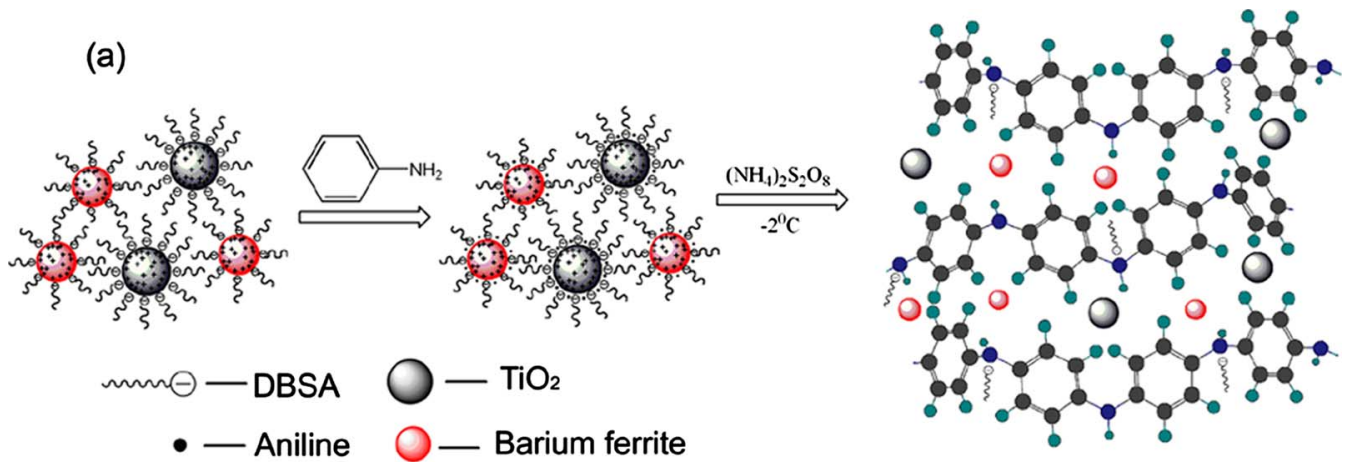

(b)

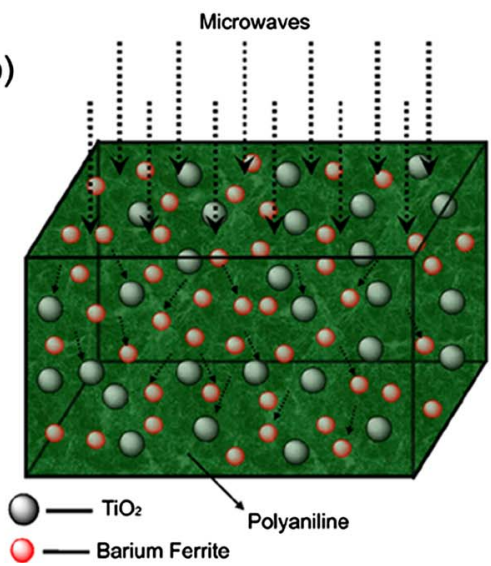

SCHEME 1. (Color online) Schematic representation of (a) polymerization of micellar solution of DBSA containing the barium ferrite and TiO ${ }_{2}$ nanoparticles using ammonium persulphate (APS) as oxidant and (b) the interaction of the microwave with the polymer composite resulting in its attenuation due to the scattering with the nanoparticles.

added to the above solution and again homogenized for $2-3 \mathrm{~h}$ to form micelles of aniline with barium ferrite and $\mathrm{TiO}_{2}$. The micelles so formed have been polymerized at $0{ }^{\circ} \mathrm{C}$ by emulsion polymerization using $\left(\mathrm{NH}_{4}\right)_{2} \mathrm{~S}_{2} \mathrm{O}_{8}(0.1 M)$ as oxidant. The product so obtained has been demulsified by treating it with equal amount of isopropyl alcohol. The precipitate so obtained were filtered out and washed with alcohol and dried at $60-65{ }^{\circ} \mathrm{C}$.

Different formulations of polymer composites have been synthesized in DBSA medium in order to check the effect of ferrite constituents on the properties. In these formulations aniline and $\mathrm{TiO}_{2}$ weight ratio was kept constant and barium ferrite ratio was varied. In formulation PBT21, aniline and $\mathrm{TiO}_{2}$ weight ratio is $1: 1$ and barium ferrite constituent is 0.5 . Similarly, in PBT11 and PBT12 compositions, aniline to ferrite to $\mathrm{TiO}_{2}$ weight ratios are in 1:1:1 and 1:2:1 proportions respectivley. Beside this, polyaniline- $\mathrm{TiO}_{2}$ (PT11) composite having monomer to $\mathrm{TiO}_{2}$ weight ratio of $1: 1$, polyanilinebarium ferrite (PF12) with monomer to ferrite weight ratio of $1: 2$, and pure polyaniline doped with DBSA (PD13) have also been synthesized for comparative study.

The particle size and the morphology of $\mathrm{TiO}_{2}$, barium ferrite, and polymer composites have been examined using transmission electron microscopy (TEM) (Phillips CM-12). The TEM samples have been prepared by dispersing the powder in isopropanol using sonification and placing small drop in the suspension on carbon coated copper grids. High resolution TEM (HRTEM) has been carried out on Technai G20-stwin $(200 \mathrm{kV})$ with point resolution of $1.44 \AA$ and line resolution of $2.32 \AA$. The presence of $\mathrm{TiO}_{2}$ and barium ferrite in the polymer composite has been confirmed by $\mathrm{X}$-ray diffraction (XRD) studies carried out on D8 Advance x-ray diffractometer (Bruker) using $\mathrm{Cu} K \alpha$ radiation $(\lambda$ $=1.540598 \AA$ ) in scattering range $(2 \theta)$ of $10^{\circ}-70^{\circ}$ with a scan rate of $0.02 \mathrm{deg} / \mathrm{s}$ and slit width of $0.1 \mathrm{~mm}$. EM shielding and dielectric measurements have been carried out on an Agilent E8362B Vector network analyzer in the microwave range of $12.4-18 \mathrm{GHz}$ ( $K_{u}$-band). Powder samples have been compressed in the form of rectangular pellets $(2 \mathrm{~mm}$ thick) and inserted in $15.8 \times 7.9 \times 6 \mathrm{~mm}^{3}$ copper sample holder connected between the waveguide flanges of network analyzer.

\section{RESULTS AND DISCUSSION}

Synthesis of polyaniline- $\mathrm{TiO}_{2}$-barium ferrite is carried out using DBSA as surfactant which also work as a dopant. Due to the hydrophilic and hydrophobic parts in DBSA, it results in the formation of micelles and when this micellar solution is polymerize with the help of oxidant, polymerization takes place at the boundaries of the micelles and nanoparticles are trapped inside the polymer chain [Scheme 1(a)]. TEM and HRTEM images of the PBT nanocomposite are shown in Fig. 1. Figure 1(a) clearly shows that when the nanoparticles of $\mathrm{TiO}_{2}$ and barium ferrite are polymerized along with aniline, they form a core-shell type of morphology. From the figure, it is also observed that an array of nanoparticles is formed during the in situ emulsion polymer- 

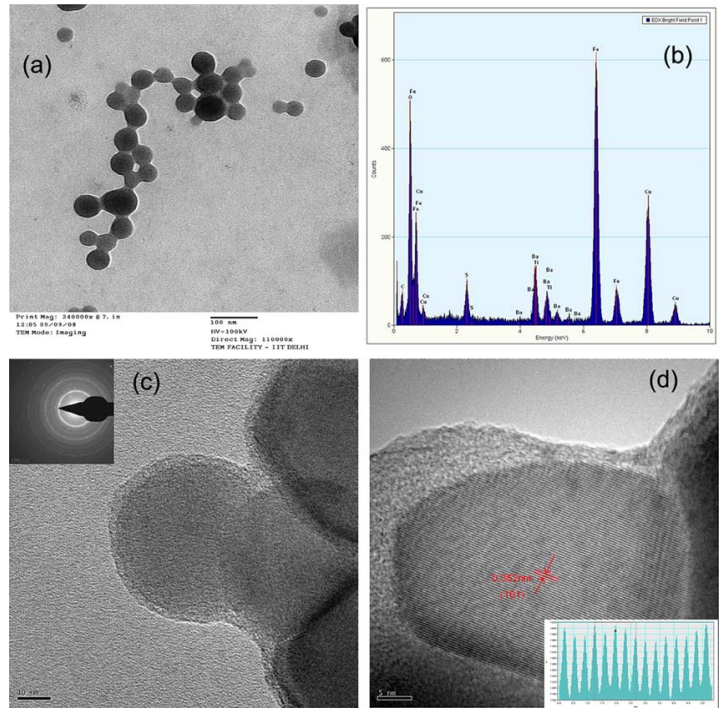

FIG. 1. (Color online) (a) Transmission electron micrograph of PBT12 nanocomposite showing the formation of array of nanoparticles via conducting matrix of polyaniline. (b) Energy dispersive x-ray pattern of PBT12 showing the approximate percentage of the element present in the nanocomposite. (c) HRTEM image of PBT21 while the inset demonstrates the electron diffraction pattern of PBT12 having ring of crystalline barium ferrite and $\mathrm{TiO}_{2}$. (d) HRTEM image, fringes indicate the presence of crystalline $\mathrm{TiO}_{2}$ particle, while the outer shell shows the polymer matrix of PBT12 nanocomposite. The inset of (d) shows the depth profile of the fringe with $d$-spacing of $0.352 \mathrm{~nm}$.

ization process and directly indicates that the particles are separated by the polymer matrix. The presence of conducting shell encapsulating the magnetic and dielectric nanoparticles is helpful in enhancing the absorption of the EM wave. HRTEM images of PBT21 [Fig. 1(c)] and PBT12 [Fig. 1(d)] also confirm the core-shell morphology of the nanocomposites. The shell of the particle gives an impression of an amorphous layer as no fringes have been observed at the shell while the $d$-spacing of the particles confirm the presence of $\mathrm{TiO}_{2}$ nanoparticle at the core. The presence of other elements in the polymer composite is confirmed by the energy dispersive x-ray spectroscopy (EDAX) studies [Fig. 1(b)].

The presence of $\mathrm{TiO}_{2}$ and barium ferrite in the nanocomposites is confirmed by XRD patterns [Fig. 2(a)]. The main peaks for $\mathrm{TiO}_{2}$ are observed at $2 \theta$ values of $25.283^{\circ}(d$ $=3.520 \AA), 37.784^{\circ}(d=2.379 \AA), 38.530^{\circ} \quad(d=2.335 \AA)$, $48.032^{\circ}(d=1.893 \AA), 53.874^{\circ}(d=1.700 \AA), 55.025^{\circ}(d$ $=1.667 \AA)$, and $62.660^{\circ}(d=1.481 \AA)$ corresponding to (101), (004), (112), (200), (105), (211), and (204) reflections [curve (a)], respectively. For barium ferrite, main peaks are observed at $2 \theta$ values of $30.294^{\circ}(d=2.9480), 32.141^{\circ}(d$ $=2.7827), \quad 34.083^{\circ} \quad(d=2.6284), \quad 37.046^{\circ} \quad(d=2.4247)$, $40.254^{\circ} \quad(d=2.2386), \quad 42.391^{\circ} \quad(d=2.1305), \quad 55.018^{\circ} \quad(d$ $=1.6677), 56.477^{\circ}(d=1.6280)$, and $63.054^{\circ}(d=1.4731)$ cor responding to the (110), (107), (114), (203), (205), (206), (217), (201), and (220) reflections [curve (f)], respectively. All the observed peaks have been matched with the standard XRD pattern of $\mathrm{TiO}_{2}{ }^{17}$ and barium ferrite. ${ }^{18}$ The peaks of barium ferrite were observed in all the compositions of polyaniline composites with $\mathrm{TiO}_{2}$ and barium ferrite, which indicates the presence of ferrite particles in the polymer matrix. The increase in the intensity of the peaks demonstrates
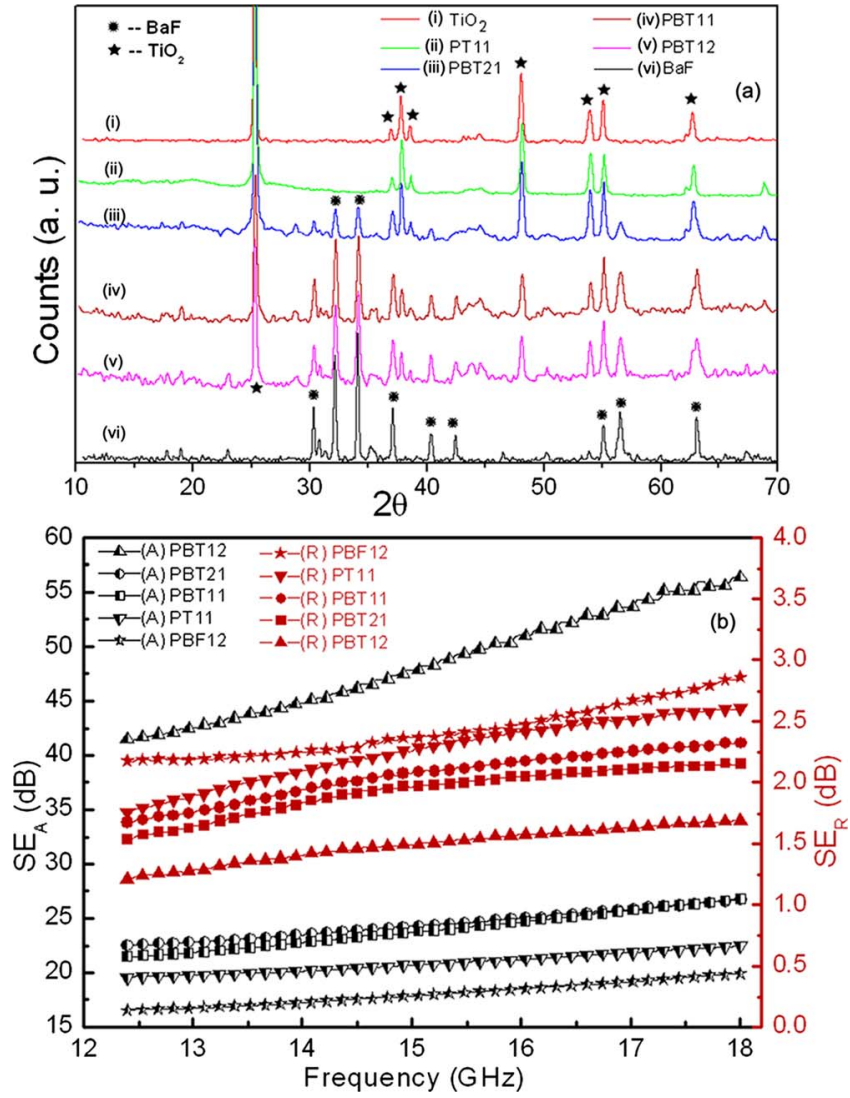

FIG. 2. (Color online) (a) XRD patterns of (i) $\mathrm{TiO}_{2}$, (ii) PT11, (iii) PBT21, (iv)PBT11, (v) PBT12, and (vi) Ba ferrite. Plots (c), (d), and (e) confirm the presence of $\mathrm{TiO}_{2}$ and barium ferrite in the polymer composite. (b) Dependence of $\mathrm{SE}$ and $\mathrm{SE}_{R}$ ) on frequency showing the effect of barium ferrite concentration on the $\mathrm{SE}_{A}$ value of the nanocomposites.

the increase in the ratio of barium ferrite. The crystallite size of barium ferrite and $\mathrm{TiO}_{2}$ has been calculated by using Scherrer's formula,

$$
D=k \lambda / \beta \cos \theta,
$$

where $\lambda$ is the $\mathrm{x}$-ray wavelength, $k$ is the shape factor, $D$ is the crystallite size for the individual peak of the crystal (in angstroms), $\theta$ is the Bragg angle (in degrees), and $\beta$ is the full width at half maxima (in radians). The value of $k$ is assigned as 0.89 , which depends on several factors including the Miller index of the reflecting plane and the shape of the crystal. The crystallite size of barium ferrite particles has been calculated using Eq. (1) and estimated to be $25 \mathrm{~nm}$, while the crystallite size of $\mathrm{TiO}_{2}$ has been found to be $36.6 \mathrm{~nm}$. The presence of peaks of $\mathrm{TiO}_{2}$ and barium ferrite shows the formation of composite having separate phases of both the compounds dispersed in the polymer matrix.

The EMI SE of a material is defined as the ratio of transmitted power to the incident power and is given by

$$
\mathrm{SE}(\mathrm{dB})=-10 \log \left(\frac{P_{T}}{P_{O}}\right),
$$

where $\mathrm{P}_{T}$ and $\mathrm{P}_{O}$ are the transmitted and incident EM powers, respectively. For a shielding material, total $\mathrm{SE}=\mathrm{SE}_{R}$ $+\mathrm{SE}_{A}+\mathrm{SE}_{M}$, where $\mathrm{SE}_{R}, \mathrm{SE}_{A}$, and $\mathrm{SE}_{M}$ are due to reflection, absorption, and multiple reflections, respectively. 
In two port network, $S$-parameters $S_{11}\left(S_{22}\right), S_{21}\left(S_{12}\right)$ represent the reflection and the transmission coefficients given as

$$
\begin{aligned}
& T=\left|\frac{E_{T}}{E_{I}}\right|^{2}=\left|S_{21}\right|^{2}=\left|S_{12}\right|^{2}, \\
& R=\left|\frac{E_{R}}{E_{I}}\right|^{2}=\left|S_{11}\right|^{2}=\left|S_{22}\right|^{2},
\end{aligned}
$$

and

$$
\text { absorption coefficient }(A)=1-R-T \text {. }
$$

Here, it is noted that the absorption coefficient is given with respect to the power of the incident EM wave. If the effect of multiple reflections between both interfaces of the material is negligible, then the relative intensity of the effective incident EM wave inside the material after reflection is based on the quantity $(1-R)$. Therefore, the effective absorbance $\left(A_{\text {eff }}\right)$ can be described as $A_{\text {eff }}=(1-R-T) /(1-R)$ with respect to the power of the effective incident EM wave inside the shielding material. It is convenient to express the reflectance and effective absorbance in the form of $-10 \log (1-R)$ and $-10 \log \left(1-A_{\text {eff }}\right)$ in decibel $(\mathrm{dB})$, respectively, ${ }^{19}$ which give $\mathrm{SE}_{R}$ and $\mathrm{SE}_{A}$ as

$$
\mathrm{SE}_{R}=-10 \log (1-R)
$$

and

$$
\mathrm{SE}_{A}=-10 \log \left(1-A_{\text {eff }}\right)=-10 \log \frac{T}{1-R} .
$$

For the material the skin depth $(\delta)$ is the distance up to which the intensity of the EM wave decrease to $1 / e$ of its original strength. The skin depth is related with the attenuation constant $(\beta)$ of the wave propagation vector $\delta=1 / \beta$ $=\sqrt{2 / \omega \mu \sigma_{\mathrm{ac}}}$ with the approximations that $\sigma \gg \omega \varepsilon$. As $\delta$ $\propto \omega^{-1 / 2}$, therefore, at low frequencies for the electrically thin samples $(d \ll \delta)$ the SE of the sample is describe as

$$
\mathrm{SE}(\mathrm{dB})=20 \log \left(1+\frac{1}{2} Z_{O} d \sigma\right),
$$

where $\sigma$ is the ac conductivity, $Z_{O}$ is free space impedance, and $d$ is the sample thickness. While for the higher frequencies, sample thickness (electrically thick samples) is sufficiently greater than skin depth and EMI SE for the plane electromagnetic wave ${ }^{20}$ is given as

$$
\begin{aligned}
& \mathrm{SE}(\mathrm{dB})=\mathrm{SE}_{R}(\mathrm{~dB})+\mathrm{SE}_{A}(\mathrm{~dB}), \\
& \mathrm{SE}_{R}(\mathrm{~dB}) \approx 10 \log \left(\frac{\sigma_{\mathrm{ac}}}{16 \omega \varepsilon_{0} u_{r}}\right),
\end{aligned}
$$

and

$$
\mathrm{SE}_{A}(\mathrm{~dB})=20 \frac{d}{\delta} \log e,
$$

where $\sigma_{\mathrm{ac}}$ depends on the dielectric properties ${ }^{21}\left(\sigma_{\mathrm{ac}}\right.$ $\left.=\omega \varepsilon_{0} \varepsilon^{\prime \prime}\right)$ of the material, $\omega$ is the angular frequency $(\omega$ $=2 \pi f), \varepsilon_{0}$ is the free space permittivity, and $\mu_{r}$ is the relative magnetic permeability of the sample. In Eq. (9), the first term is related to the reflection of the EM wave and contributes as the SE due to reflection. The second term expresses the loss due to the absorption of the wave when it passes trough the shielding material. In microwave range, the contribution of the second part becomes more as compared to the reflection term.

In metallic materials, the SE is very high and mainly attributed to the reflection of the EM radiation due to its high conductivity, whereas in the case of conducting polymers having moderate conductivity the contribution to the SE comes from both the reflection and the absorption. It has been observed that conducting ferromagnetic composites of polyaniline with barium ferrite and $\mathrm{TiO}_{2}$ have SE mainly due to absorption. Figure 2(b) shows the variation in the SE with frequency in the $12.4-18 \mathrm{GHz}$ range. As seen in the figure, PBF12 shows the $\mathrm{SE}_{A}$ value of $19 \mathrm{~dB}$, while for PT11 nanocomposite, the $\mathrm{SE}_{A}$ value of $22 \mathrm{~dB}$ is observed. With the addition of barium ferrite nanoparticles in the ratios of 2:1 and 1:1, an increase in the microwave absorption is nominal $(\sim 3 \mathrm{~dB})$, but when double amount of barium ferrite is taken as compared to $\mathrm{TiO}_{2}$ (sample PBT12), substantial enhancement in the absorption of EM radiation is observed. The maximum SE of $58 \mathrm{~dB}$ has been achieved for the PBT12 sample having the polymer: $\mathrm{TiO}_{2}$ :ferrite ratio of $1: 1: 2$. It is observed that the SE increases with the increase in the ferrite concentration and with the increase in frequency. The increase in the absorption part is mainly attributed to the presence of a high dielectric constant material and a magnetic material which increase more scattering, which, in turn, results in more attenuation of the EM radiations [Scheme $1(b)]$.

To investigate the possible mechanism and effects giving rise to the improvement in microwave absorption, complex permittivity $\left(\varepsilon_{r}=\varepsilon^{\prime}-j \varepsilon^{\prime \prime}\right)$, and permeability $\left(\mu_{r}=\mu^{\prime}-j \mu^{\prime \prime}\right)$ of the samples were measured. The real $\left(\varepsilon^{\prime}\right)$ and imaginary $\left(\varepsilon^{\prime \prime}\right)$ parts of complex permittivity versus frequency are shown in Figs. 3(a) and 3(b). The real part $\left(\varepsilon^{\prime}\right)$ is mainly associated with the amount of polarization occurring in the material and the imaginary part $\left(\varepsilon^{\prime \prime}\right)$ is related to the dissipation of energy. In polyaniline, strong polarization occurs due to the presence of polaron/bipolaron and other bound charges which leads to high value of $\varepsilon^{\prime}$ and $\varepsilon^{\prime \prime}$. With the increase in frequency, the dipoles present in the system cannot reorient themselves along with the applied electric field, as a result of which dielectric constant decreases. The main characteristic feature of $\mathrm{TiO}_{2}$ is that it has high dielectric constant with dominant dipolar polarization and the associated relaxation phenomenon constitutes the loss mechanism. ${ }^{22}$ With the addition of barium ferrite and $\mathrm{TiO}_{2}$ in polyaniline matrix, significant increase in the imaginary part of complex permittivity has been observed. The higher values of the dielectric loss is attributed to the more interfacial polarization due to the presence of insulating barium ferrite particles and high dielectric $\mathrm{TiO}_{2}$ particles which consequently lead to more SE due to absorption.

Figures 3(c) and 3(d) show the variation in the real part and imaginary part of magnetic permeability with frequency. The magnetic permeability of all the samples decreases with the increase in frequency, whereas higher magnetic loss has been observed for higher percentage of barium ferrite 

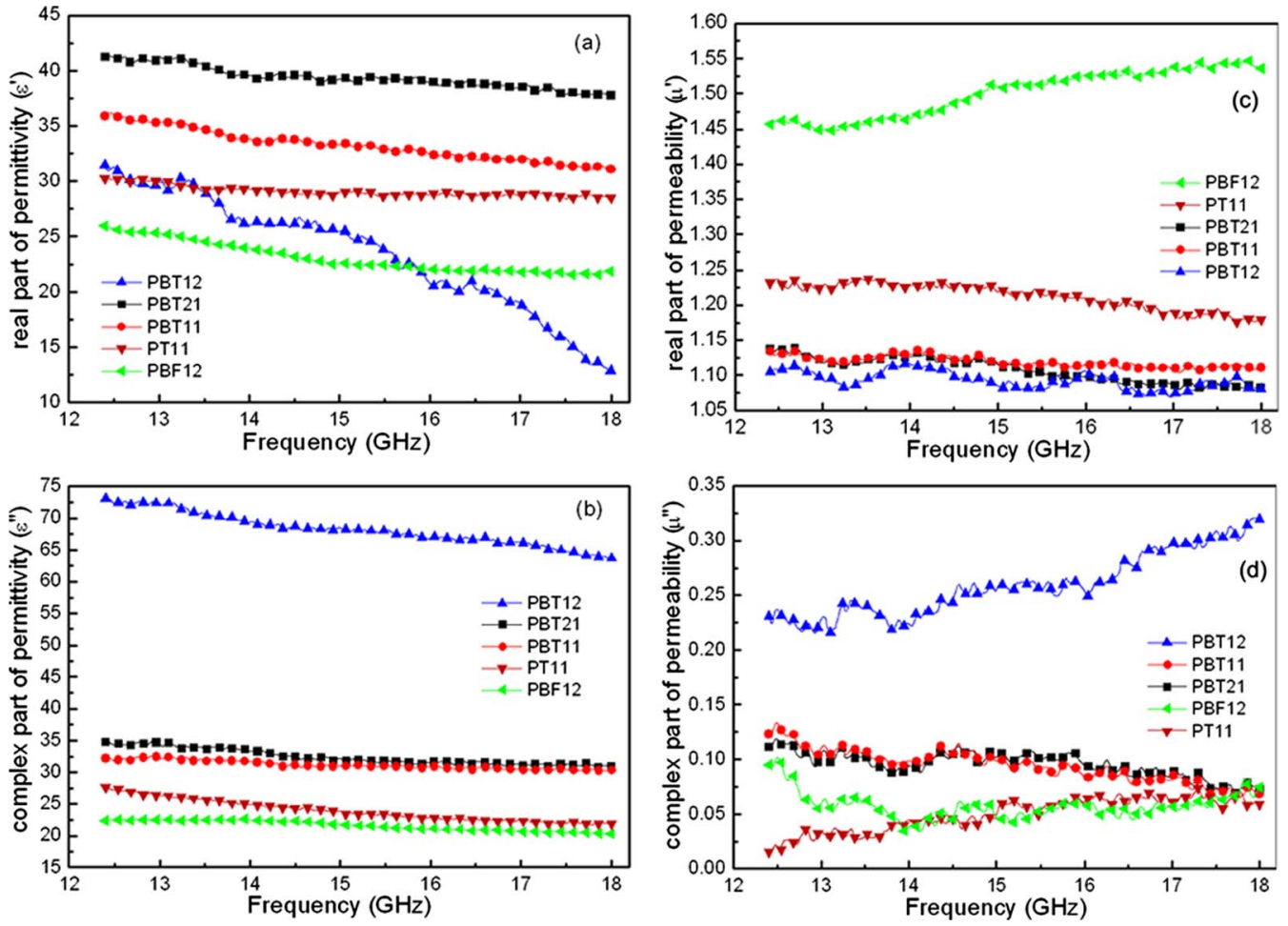

FIG. 3. (Color online) (a) Behavior of (a) real and (b) imaginary parts of permittivity. (c) and (d) show the variation in real and imaginary parts of magnetic

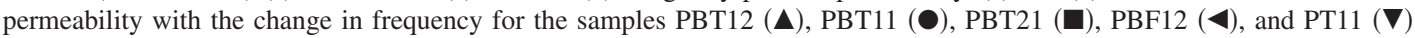

(PBT12) in the polymer matrix. The magnetic loss is caused by the time lag of magnetization vector $(M)$ behind the magnetic field vector. The change in magnetization vector is generally brought about by the rotation of magnetization and the domain wall displacement. These motions lag behind the change in the magnetic field and contribute to the magnetic loss $\left(\mu^{\prime \prime}\right)$. The rotation of the domain of magnetic nanoparticles might become difficult due to the effective anisotropy (magnetocrystalline anisotropy and shape anisotropy). The surface area, number of atoms with dangling bonds, and unsaturated coordination on the surface of polymer matrix are all enhanced. These variations lead to the interface polarization and multiple scattering, which is useful for the absorption of large number of microwaves. ${ }^{23}$

Figure 4(a) (inset) shows the variation in $\sigma_{\mathrm{ac}}$ with the frequency for the sample PBT11, calculated from the dielectric measurements $\left(\sigma_{\mathrm{ac}}=\omega \varepsilon_{0} \varepsilon^{\prime \prime}\right)$. To relate $\sigma_{\mathrm{ac}}$ to the shielding parameters of the material, $\mathrm{SE}_{R}$ is plotted against $\log \sigma_{\mathrm{ac}}$ [Fig. 4(a)]. Higher value of conductivity is required for high SE due to reflection. For the absorption part, the skin depth of the samples has been calculated using the relation, $\delta$ $=\sqrt{2 / \omega \mu \sigma_{\mathrm{ac}}}$ and its variation with frequency is shown in Fig. 4(b) (inset). It has been observed that the skin depth decreases with frequency, which demonstrates that mainly surface conduction exists at the higher frequencies. The dependence of skin depth on the conductivity and magnetic permeability reveal that for highly conducting and magnetic material, the skin depth is very small. From Eq. (11), better $\mathrm{SE}_{A}$ can be achieved from the highly conducting and magnetic materials. The dependence of $\mathrm{SE}_{A}$ on $\left(\sigma_{\mathrm{ac}}\right)^{1 / 2}$ is shown in Fig. 4(b).
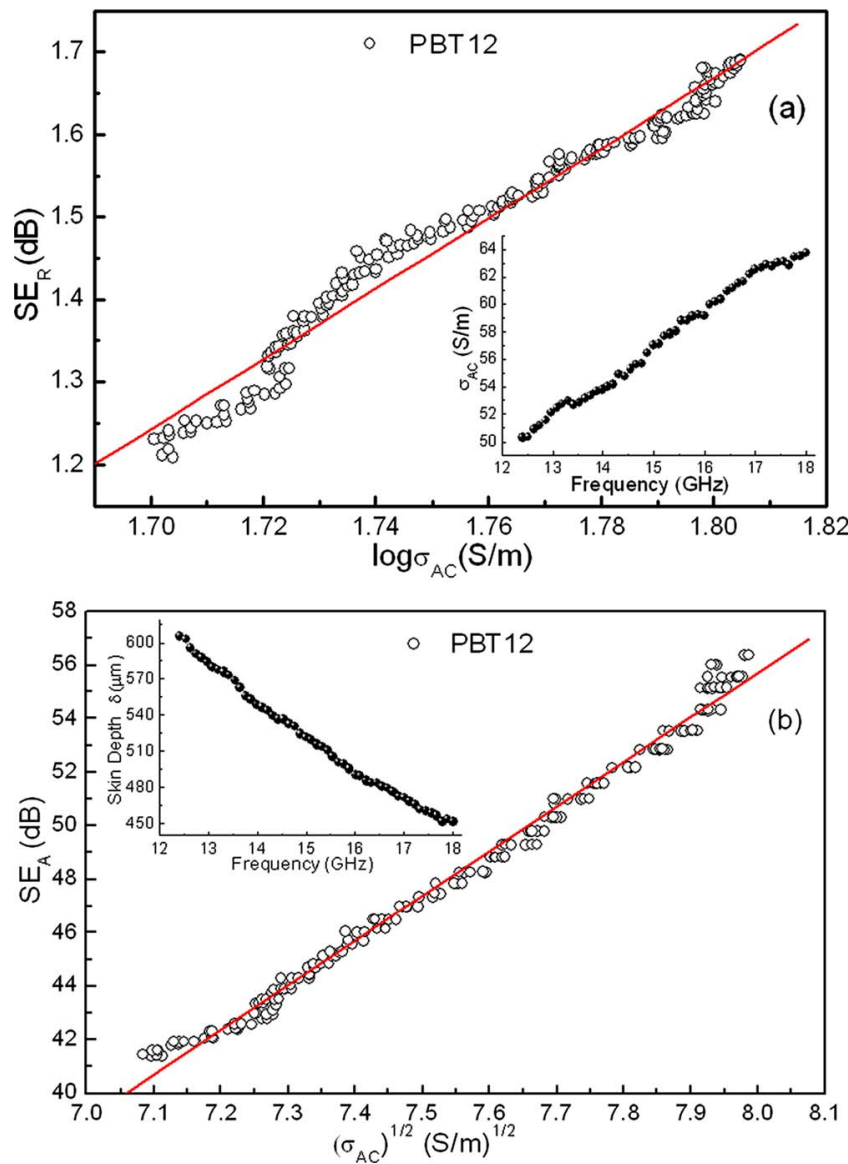

FIG. 4. (Color online) (a) Variation in $\mathrm{SE}_{R}$ as a function of $\log \sigma_{\mathrm{ac}}$, while the inset shows the variation in $\sigma_{\mathrm{ac}}$ with the increase in frequency. (b) Variation in $\mathrm{SE}_{A}$ as a function of $\left(\sigma_{\mathrm{ac}}\right)^{1 / 2}$, while the inset shows the change in skin depth $(\delta)$ with the increase in frequency for the PBT12 sample. 


\section{CONCLUSIONS}

In conclusion, polyaniline- $\mathrm{TiO}_{2}$-barium ferrite nanocomposites, prepared by the microemulsion method, have excellent microwave absorption properties. The microwave absorption property of the composites strongly depends on the intrinsic properties of barium ferrite and $\mathrm{TiO}_{2}$ nanoparticles in the polymer matrix. The incorporation of $\mathrm{TiO}_{2}$ and barium ferrite results in the formation of array of nanoparticles, which leads to more interfacial dipolar polarization and higher anisotropic energy due to the nanosize that consequently contributes to the high values of SE due to absorption. The dependence of $\mathrm{SE}_{A}$ on the magnetic permeability and ac conductivity shows that better absorption value has been obtained for material with higher conductivity and magnetization.

\section{ACKNOWLEDGMENTS}

The authors wish to thank Director N.P.L for his keen interest in the work. The authors also thank Dr. Rashmi for recording XRD pattern of the samples. A.O. is thankful to CSIR for providing the necessary fellowship.

${ }^{1}$ Y. K. Hong, C. Y. Lee, C. K. Jeong, D. E. Lee, K. Kim, and J. Joo, Rev. Sci. Instrum. 74, 1098 (2003).

${ }^{2}$ H. K. Kim, M. S. Kim, K. Song, Y. H. Park, S. H. Kim, J. Joo, and J. Y. Lee, Synth. Met. 135, 105 (2003).

${ }^{3}$ N. H. Hoang, J. L. Wojkiewicz, J. L. Miane, and R. S. Biscarro, Polym.
Adv. Technol. 18, 257 (2007).

${ }^{4}$ Y. Wang and X. Jing, Polym. Adv. Technol. 16, 344 (2005).

${ }^{5}$ S. K. Dhawan, N. Singh, and S. Venkatachalam, Synth. Met. 129, 261 (2002).

${ }^{6}$ N. C. Das, S. Yamazaki, M. Hikosaka, T. K. Chaki, D. Khastgir, and A. Chakraborty, Polym. Int. 54, 256 (2005).

${ }^{7}$ H. M. Kim, K. Kim, C. Y. Lee, J. Joo, S. J. Cho, H. S. Yoon, D. A. Pejakovic, J. W. Yoo, and A. J. Epstein, Appl. Phys. Lett. 84, 589 (2004).

${ }^{8}$ Y. Huang, N. Li, Y. Ma, F. Du, F. Li, X. He, X. Lin, H. Gao, and Y. Chen, Carbon 45, 1614 (2007).

${ }^{9}$ K. Y. Park, S. E. Lee, C. G. Kim, and J. H. Han, Compos. Struct. 81, 401 (2007).

${ }^{10}$ P. Chandrasekhar and K. Naishadham, Synth. Met. 105, 115 (1999).

${ }^{11}$ J. L. Wallace, IEEE Trans. Magn. 29, 4209 (1993).

${ }^{12}$ B. W. Li, Y. Shen, Z. X. Yue, and C. W. Nan, J. Magn. Magn. Mater. 313, 322 (2007).

${ }^{13}$ J. R. Liu, M. Itoh, and K. Machida, Appl. Phys. Lett. 83, 4017 (2003).

${ }^{14}$ R. Che, L. M. Peng, X. Duan, Q. Chen, and X. Liang, Adv. Mater. (Weinheim, Ger.) 16, 401 (2004).

${ }^{15}$ S. M. Abbas, R. Chatterjee, A. K. Dixit, A. V. R. Kumar, and T. C. Goel, J. Appl. Phys. 101, 074105 (2007).

${ }^{16}$ J. Qiu, H. Shen, and M. Gu, Powder Technol. 154, 116 (2005).

${ }^{17}$ JCPDS Card No. 84-1285.

${ }^{18}$ JCPDS Card No. $39-1433$.

${ }^{19}$ K. Singh, A. Ohlan, P. Saini, and S. K. Dhawan, Polym. Adv. Technol. 19, 229 (2008)

${ }^{20}$ N. F. Colaneri and L. W. Shacklette, IEEE Trans. Instrum. Meas. 41, 291 (1992).

${ }^{21}$ R. Singh, J. Kumar, R. K. Singh, R. C. Rastogi, and V. Kumar, New J. Phys. 9, 40 (2007).

${ }^{22}$ D. L. Leslie-Pelecky and R. D. Rieke, Chem. Mater. 8, 1770 (1996).

${ }^{23}$ X. F. Zhang, X. L. Dong, H. Huang, Y. Y. Liu, W. N. Wang, X. G. Zhu, B. Lv, J. P. Lei, and C. G. Lee, Appl. Phys. Lett. 89, 053115 (2006). 